\title{
LAS MUJERES SOMETIDAS A REDUCCIÓN MAMARIA MEJORAN SU APARIENCIA ESTÉTICA, AUTOESTIMA, SÍNTOMAS FÍSICOS Y VIDA SEXUAL*
}

\author{
Drs. Stefan Danilla E. ${ }^{1}$, Pedro Cuevas T. ${ }^{1}$, Marco Ríos V. ${ }^{1}$, Rocío Jara C. ${ }^{1}$, \\ Omar Chávez M. ${ }^{1}$, Estefanía Enríquez Chiang. ${ }^{1}$, Rolando Schulz T. ${ }^{1}$, \\ Cristian Erazo C. ${ }^{1}$, Susana Benítez S. ${ }^{1}$, Patricio Andrades C. ${ }^{1}$, Sergio Sepúlveda P. ${ }^{1}$ \\ 1 Departamento de Cirugía Hospital Clínico de la Universidad de Chile. \\ Santiago, Chile.
}

\begin{abstract}
Effects of breast reduction surgery on the quality life

Background: The results of breast reduction surgery are usually reported in terms of anthropometric assessments. However the impact of surgery in quality of life is seldom informed. Aim: To assess the effects of breast reduction surgery on the quality of life of patients. Material and Methods: The "Breast-Q Reduction and Mastopexy Module" was prospectively applied to all women subjected to breast reduction surgery in a University Hospital during one year. Results: Twenty women answered the survey in the preoperative period and 20 in the postoperative period. The most commonly cutaneous pattern employed that the inverted $\mathrm{T}$ in 10 women, followed by the Wise pattern in six patients. The most commonly used pedicles were superior in 10 patients, followed by superomedial in seven patients. Mean excision weight was $923 \mathrm{~g}$ (range 150-2850). Mean Breast Q score improved from 45 to $65 \%$. Satisfaction with breast appearance improved from 31 to $83 \%$, self-esteem from 39 to $80 \%$, sexuality from 50 to $81 \%$ and physical symptoms decreased from 48 to $16 \%$. There was a significant association between the magnitude of excision and improvement in physical symptoms. Conclusions: Quality of life improves notably in women after breast reduction surgery.
\end{abstract}

Key words: Breast reduction, quality of life, surgical technique.

\section{Resumen}

Objetivo: Tradicionalmente la evaluación de resultados en reducción mamaria se ha limitado a evaluaciones antropométricas, que si bien nos entregan una evaluación objetiva de un aspecto de los resultados, resultan insuficientes para evaluar el impacto global en calidad de vida $(\mathrm{CdV})$ de la cirugía en nuestros

*Recibido el 5 de diciembre de 2014 y aceptado para publicación el 12 de julio de 2015.

Los autores no refieren conflictos de interés.

Aprobado por el Comité de Ética de la Universidad de Chile (Acta $\left.N^{\circ} 22\right)$ y realizado bajo los principios éticos propuestos en la declaración de Helsinki.

Correspondencia: Dr. Stefan Danilla E.

drstefandanilla@gmail.com 
pacientes. Es por esto que el objetivo de este trabajo es determinar el cambio en calidad de vida en mujeres sometidas a reducción mamaria y explorar sus factores determinantes. Materiales y Métodos: Se realizó un estudio de cohorte prospectiva con todas las pacientes que ingresaron al Hospital Clínico de la Universidad de Chile (HCUCH) y se sometieron a reducción mamaria por cualquier técnica desde julio de 2013 a abril de 2014. La calidad de vida (CdV) se midió con el instrumento validado Breast-Q Reduction and Mastopexy Module. Se utilizó estadística descriptiva para caracterizar la muestra y analítica para determinar el cambio en CdV y sus factores pronósticos. Resultados: Entre julio de 2013 y abril de 2014 se operaron 25 pacientes, el seguimiento promedio fue de 7 meses. Veinte pacientes contestaron el Breast- $Q$ preoperatorio y 20 el postoperatorio. El patrón cutáneo más empleado fue la T acortada $(10 ; 55,6 \%)$ seguido por el patrón de Wise (6; $33,3 \%)$; el pedículo más usado fue el superior $(10 ; 55,6 \%)$ seguido del superomedial $(7 ; 38,9 \%)$. La resección promedio total fue de $923 \mathrm{~g}$, variando entre $150 \mathrm{~g}$ y $2.850 \mathrm{~g}$. El puntaje promedio del Breast- $Q$ subió de $45 \%$ a $65 \%(\mathrm{p}=0,005)$. Las pacientes mejoraron su satisfacción con la apariencia mamaria desde un $30,8 \%$ a un $82,9 \%(\mathrm{p}<0,001)$, su autoestima de $38,7 \%$ a $80,1 \%(\mathrm{p}<0,001)$, síntomas físicos de $47,8 \%$ a $15,5 \%$ (p $=0,003)$ y sexualidad de $50,0 \%$ a $81,4 \%(\mathrm{p}<0,001)$. La magnitud de la resección se asoció a la mejoría de síntomas físicos (Coeff 0,$07 ; p=0,023$ ). No se encontraron otras asociaciones significativas entre el cambio en parámetros de calidad de vida y variables independientes. Conclusiones: En este reporte preliminar hemos podido objetivar que existe una mejoría significativa en la calidad de vida de las pacientes sometidas a una reducción mamaria tanto en forma global como también en cada uno de los aspectos evaluados, mejorando significativamente su autoimagen, autoestima, vida sexual y síntomas físicos.

Palabras clave: Reducción mamaria, Breast- $Q$, calidad de vida.

\section{Introducción}

La cirugía de reducción mamaria constituye uno de los motivos más frecuentes de consulta en cirugía plástica. Según cifras de la Sociedad Americana de Cirugía Plástica, en Estados Unidos, se realizan alrededor de 100.000 reducciones mamarias al año ${ }^{1}$. Tradicionalmente la evaluación de resultados se ha enfocado en la antropometría postoperatoria, a lo cual se han agregado avances en imagenología, específicamente la fotografía 3D que ha demostrado ser una herramienta útil, que permite realizar evaluaciones volumétricas pre y postoperatorias, cuantificar cambios en forma y realizar planificación preoperatoria certera ${ }^{2-4}$. Si bien todos estos aspectos constituyen un avance importante, siguen centrándose en la perspectiva del cirujano, dejando de lado la perspectiva del paciente, pasando por alto aspectos relevantes de medir, tales como síntomas físicos, impacto en vida sexual, relaciones sociales y autoimagen. Dichos aspectos impactan significativamente en la $\mathrm{CdV}$ de los pacientes, es por esto que a fin de obtener una evaluación global de los resultados y con el objetivo de cuantificar el impacto en $\mathrm{CdV}$ se torna necesario la utilización de instrumentos de medición de resultados desde la perspectiva del paciente (PROM, por su sigla en inglés), que hayan sido validados para su uso en nuestro medio. Hasta hace poco no existían instrumentos que permitieran evaluar de forma objetiva y comparable el impacto en calidad de vida (CdV) de los resultados en reducción mamaria. Con el fin de poder estandarizar la evaluación de resultados nuestro grupo ha validado el instrumento Breast $Q$
Reduction and Mastopexy, (Breast-Q) Module para uso en Chile 5 . Este instrumento consta de 4 módulos de evaluación divididos en 39 ítems que corresponden a: autoimagen corporal, desempeño socialautoestima, sexualidad y síntomas físicos. Debido a sus propiedades psicométricas el instrumento proporciona una escala objetiva y reproducible para evaluar los resultados quirúrgicos en cirugía de reducción mamaria ${ }^{6}$. El objetivo del presente estudio es evaluar el cambio en calidad de vida en mujeres sometidas a reducción mamaria y explorar sus factores determinantes.

\section{Material y Método}

\section{Diseño}

Para lograr nuestro objetivo diseñamos un estudio de cohorte prospectivo, llevado a cabo en un hospital universitario tipo I; se incorporaron al estudio todas las pacientes sometidas a reducción mamaria por cualquier técnica desde julio de 2013 a abril de 2014 y que consintieron participar del estudio. Los criterios para cirugía son mujeres en categoría ASA 1 y 2, con mamografía y ecografía mamaria BIRADS 1 o 2 y exámenes preoperatorios normales. Este estudio fue aprobado por el Comité de Ética de la Universidad de Chile (Acta $\mathrm{N}^{\mathrm{o}} 22$ ) y realizado bajo los principios éticos propuestos en la declaración de Helsinki

\section{Mediciones}

La variable principal de resultado es el cambio en calidad de vida en mujeres sometidas a reducción 
mamaria medida por el puntaje del instrumento BREAST-Q Reduction and Mastopexy Module (Tabla 1) para mamoplastía de reducción. Se consideró como mejoría la diferencia entre el puntaje preoperatorio y el postoperatorio en forma total y por dominios. Como variables pronósticas se estudiaron características demográficas, variables antropométricas y variables relativas a la cirugía. La confiabilidad de la escala alcanza el 97\% tal como se publicó por nuestro grupo en un artículo previo ${ }^{5}$.

\section{Estadística}

Se utilizó estadística descriptiva para resumir y explicar variables. Para el análisis exploratorio, la búsqueda de factores que incidieron en el cambio en calidad de vida se realizó sobre la diferencia entre el puntaje postoperatorio y el preoperatorio del Breast $Q$ utilizando esta diferencia como variable dependiente y las características demográficas, antropométricas y quirúrgicas como independientes en modelos de regresión lineal (previa normalización) y logística (ordinal y binaria).

\section{Resultados}

Entre julio de 2013 y abril de 2014 se operaron 25 pacientes. En la Tabla 2 se detallan las características generales de la población estudiada. En general se trata de mujeres jóvenes con sobrepeso y sin patología mamaria asociada. En las Tablas 3 y 4 se presentan las características antropométricas de las pacientes, el grado de ptosis y el diagnóstico preoperatorio respectivamente. Como se aprecia se trata de pacientes con ptosis e hipertrofia mamaria moderada a severa.

El seguimiento promedio fue de 7 meses. Veinte pacientes contestaron el Breast- $Q$ preoperatorio y 20 el postoperatorio durante el período de seguimiento.

El patrón cutáneo más empleado fue la $\mathrm{T}$ acortada $(10 ; 55,6 \%)$ seguido por el patrón de Wise $(6$; $33,3 \%)$; el pedículo más usado fue el superior (10; $55,6 \%)$ seguido del superomedial $(7 ; 38,9 \%)$. La resección promedio total fue de $923 \mathrm{~g}$, variando entre $150 \mathrm{~g}$ y $2.850 \mathrm{~g}$ como se aprecia en la Tabla 5.

La CdV global se elevó significativamente en el postoperatorio, tal como lo refleja el puntaje promedio del Breast- $Q$ que subió de un $45 \%$ a un $65 \%(\mathrm{p}=0,005)$. Además hay una mejoría estadísticamente significativa en cada uno de los dominios evaluados. Como se observa en la Figura 1, las pacientes mejoraron su satisfacción con la apariencia mamaria desde un $30,8 \%$ a un $82,9 \%$ ( $p<0,001)$, su autoestima de un $38,7 \%$ a un $80,1 \%(\mathrm{p}<0,001)$, síntomas físicos de un $47,8 \%$ a un $15,5 \%(\mathrm{p}=0,003)$ y su sexualidad de un $50,0 \%$ a un $81,4 \%(\mathrm{p}<0,001)$.

De todas las variables quirúrgicas evaluadas la magnitud de la resección fue la única variable que se asoció a la mejoría de síntomas físicos (Coeficiente $0,07, p=0,023)$. No se encontraron otras asociaciones significativas entre el cambio en parámetros de calidad de vida y variables independientes.

\section{Conclusiones}

Nuestros resultados demuestran de manera objetiva una mejoría significativa en la $\mathrm{CdV}$ global de los pacientes sometidos a reducción mamaria,

Tabla 1. Breast- $Q{ }^{\circledR}$ Reduction and Mastopexy Module*

\begin{tabular}{|lc|}
\hline Dominio & Ítems \\
\hline 1. Autoimagen corporal & 11 \\
2. Desempeño social-autoestima & 9 \\
3. Síntomas físicos & 14 \\
4. Sexualidad & 5 \\
\hline
\end{tabular}

Dominios y número de ítems por dominio para evaluación de resultados en mamoplastía de reducción.

Tabla 2. Características de la población estudiada $(\mathrm{n}=\mathbf{2 5})$

\begin{tabular}{|lcccc|}
\hline & Promedio & (DS) & Min & Max \\
Edad (años) & 36 & $( \pm 11,1)$ & 15 & 63 \\
Peso $(\mathrm{kg})$ & 72,6 & $( \pm 8,56)$ & 60 & 79 \\
Talla $(\mathrm{m})$ & 1,61 & $( \pm 5,29)$ & 1,50 & 1,68 \\
IMC $\left(\mathrm{kg} / \mathrm{m}^{2}\right)$ & 27,9 & $( \pm 1,59)$ & 25,7 & 30,5 \\
\hline
\end{tabular}

Tabla 3. Medidas antropométricas de las mamas (en cm)

\begin{tabular}{|lcccccc|}
\hline & Promedio (I/D*) & $\begin{array}{c}\text { Preop } \\
\text { Min }\end{array}$ & Max & Promedio (I/D) & $\begin{array}{c}\text { Postop } \\
\text { Min }\end{array}$ & Max \\
Clavícula-pezón & $28,7 / 28,5$ & $23 / 22$ & $35 / 35$ & $20,5 / 20,5$ & $17 / 17$ & $22 / 22$ \\
Pezón-surco & $14,4 / 14,3$ & $9 / 8$ & $22 / 22$ & $7,5 / 7,5$ & $6 / 6$ & $9 / 9$ \\
\hline
\end{tabular}

*I/D: mama izquierda/ mama derecha. 
Tabla 4. Diagnóstico y grado de ptosis mamaria (Grados I, II y III)

\begin{tabular}{|lrrlll|}
\hline Diagnóstico Preop & n & $\mathbf{( \% )}$ & Ptosis & n & $(\%)$ \\
Ptosis mamaria & 2 & 13,3 & Grado I & 3 & 17,6 \\
Hipertrofia mamaria & 2 & 13,3 & Grado II & 4 & 23,5 \\
Ambos & 11 & 73,3 & Grado III & 9 & 52,9 \\
& & & Sin ptosis & 1 & 5,88 \\
\hline
\end{tabular}

Tabla 5. Características de la cirugía

\begin{tabular}{|lccc|}
\hline Patrón cutáneo & n & $\mathbf{( \% )}$ & \\
T acortada & 10 & 55,6 & \\
Wise & 6 & 33,3 & \\
Circumvertical & 1 & 5,5 & \\
Periareolar & 1 & 5,5 & \\
Pedículo & $\mathbf{n}$ & $\mathbf{( \% )}$ & \\
Superior & 10 & 55,6 & \\
Superomedial & 7 & 38,8 & \\
Bipediculado & 1 & 5,56 & \\
Resección & Promedio & Min & Max \\
Derecha & 435 & 50 & 1.600 \\
Izquierda & 427 & 100 & 1.250 \\
Total & 923 & 150 & 2.850 \\
\hline
\end{tabular}

así como una mejoría significativa en cada uno de los aspectos evaluados: autoimagen, autoestima, vida sexual y síntomas físicos. La magnitud de la resección de tejido mamario se asoció de manera significativa con la mejoría de los síntomas y CdV. No hubo otras asociaciones de relevancia dentro de las variables estudiadas.

\section{Discusión}

La aplicación rigurosa de instrumentos objetivos de evaluación de resultados desde la perspectiva del paciente, constituye un campo en amplio desarrollo y de gran impacto en la investigación médica actual ${ }^{8}$. Las evaluaciones antropométricas así como los avances en imagenología tridimensional han permitido realizar planificaciones quirúrgicas más certeras, medir de manera objetiva los cambios de volumen $\mathrm{y}$ forma en el postoperatorio ${ }^{3,4}$, predecir resultados en reconstrucción mamaria o cirugías de aumento o reducción mamaria, todo lo cual se constituye en una herramienta muy útil para demostrar y comparar resultados de manera sistemática y reproducible ${ }^{5}$. Sin embargo, estos avances resultan insuficientes ya que no consideran la perspectiva del paciente, no pudiendo de esta forma cuantificar el impacto en CdV de la cirugía. Para este fin se requiere de la aplicación de un PROM. Dichos instrumentos y su aplicación nos permiten evaluar de forma objetiva y reproducible los resultados de distintas técnicas quirúrgicas, así como también nos proporcionan parámetros objetivos para demostrar los cambios y mejorías en calidad de vida ${ }^{9}$ reforzando la sensación de bienestar no sólo físico sino también en un plano emocional y social ya que consideran la tradicional perspectiva del cirujano e incorporan la perspectiva del paciente ${ }^{10}$.

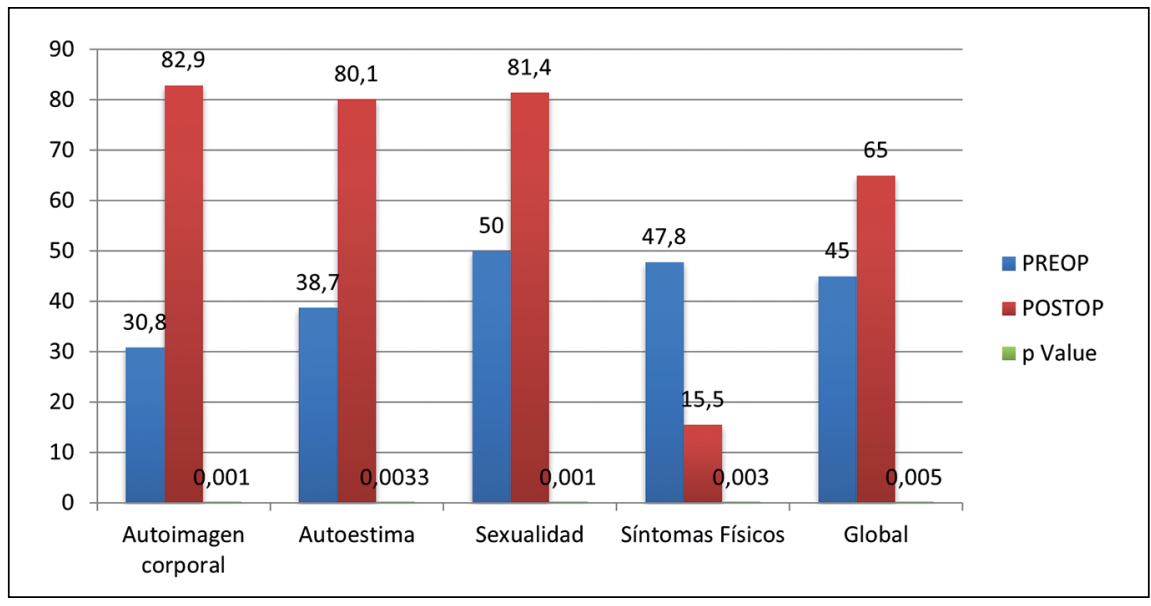

Figura 1. Porcentaje del máximo teórico pre y postoperatorio. En todos los dominios a mayor puntaje mejor calidad de vida, excepto en síntomas físicos donde a mayor puntaje, peor sintomatología. 
Los resultados preliminares de nuestro estudio demuestran una mejoría objetiva y significativa en la calidad de vida global de las pacientes sometidas a reducción mamaria independiente del estadio de su patología. Dicha mejoría abarca no sólo aspectos físicos sino también psicológicos y sociales de las pacientes intervenidas, por lo cual resulta un elemento muy útil a la hora de definir políticas de salud que tengan un impacto real en los pacientes. Estimamos que lo anterior constituye un elemento importante a considerar por las aseguradoras de salud a fin de lograr una cobertura adecuada sobre esta intervención quirúrgica, debido a su alto impacto en la Cdv de sus afiliados.

En este reporte preliminar la única variable de la técnica quirúrgica que se asoció a una mejoría en $\mathrm{CdV}$ fue el monto de tejido resecado, sin embargo, cabe señalar que se requiere un estudio con una muestra mayor a fin de determinar si otros factores técnicos pueden tener impacto significativo en los resultados.

Por lo anterior creemos necesario masificar el uso de los instrumentos validados de evaluación de resultados desde la perspectiva del paciente, permitiendo así la realización de estudios de evaluación de resultado y estudios comparativos que sin lugar a dudas contribuirán al avance científico en cirugía plástica.

\section{Referencias}

1. American Society of Plastic Surgeons 2013. Plastic Surgery Statistics Report in: http:// www.plasticsurgery. org/documents/news-resources/statistics/plastic-surgerystatistics-full-report-2013.

2. Cuevas P, Calderón M, Erazo C, Benítez S, Andrades P, Sepúlveda S, y cols. Mamoplastía de reducción: resultados desde la perspectiva del paciente. Validación lingüística y psicométrica del Breast Q Reduction and Mastopexy Module Instrument. Rev Chil Cir. 2013;65:146-9.

3. Galdino G, Nahabedian M, Chiaramonte M, Geng JZ, Klatsky S, Manson P. Clinical applications of three-dimensional photography in breast surgery. Plast Reconstr Surg. 2002; 110:58-70.

4. Tepper O, Unger J, Smll K, Feldman D, Kumar N, Choi $\mathrm{M}$, et al. Mammometrics: the standardization of aesthetic and reconstructive breast surgery. Plast Reconstr Surg. 2010; 125:393-400.

5. Donfrancesco A, Montemurro P, Heden P. Threedimensional simulated images in breast augmentation surgery: an investigation of patients satisfaction and the correlation between prediction and actual outcome. Plast Reconstr Surg. 2013;132:810-22.

6. Pusic A, Klassen A, Scott A, Klok J, Cordeiro P, Cano S. Development of a new patient reported outcome measure for breast surgery: The BREAST-Q. Plast Reconstr Surg. 2009;124:345-53.

7. World Medical Association. World Medical Association Declaration of Helsinki: Ethical principles for medical research involving human subjects. JAMA. 2013;310:2191-4.

8. Cano SJ, Browne JP, Lamping DL. Patient-based measures of outcome in plastic surgery: Current approaches and future directions. Br J Plast Surg. 2004;57:11.

9. Patel KM, Hannan CM, Gatti ME, Nahabedian MY. A head to-head comparison of quality of life and aesthetic outcomes following immediate, staged-immediate, and delayed oncoplastic reduction mammaplasty. Plast Reconstr Surg. 2011;127:2167-75.

10. Faria F, Guthrie E, Bradbury E, Brain AN. Psychosocial outcome and patient satisfaction following breast reduction surgery. Br J Plast Surg. 1999;52:448-52. 